\title{
Exploring rural palliative care patients' experiences of accessing psychosocial support through telehealth: A longitudinal approach
}

\author{
Rachel J. Rahman, ${ }^{1}$ Joseph R. Keenan, ${ }^{2}$ Joanne Hudson ${ }^{3}$ \\ ${ }^{1}$ Psychology Department, Aberystwyth University, ${ }^{2}$ Manchester Metropolitan University; ${ }^{3}$ Swansea University, UK
}

\begin{abstract}
In this research, we explore the experiences of rural palliative care patients receiving psychosocial support through telehealth. A longitudinal approach considered how experiences vary over time. Three patients with a terminal cancer diagnosis were given a laptop to access psychosocial support via telehealth over three months. Semi-structured interviews were conducted at monthly intervals. Interpretative phenomenological analysis identified four themes: Deepening understanding through unburdened and continuous connections; the ever-present paradox of visible and invisible telehealth; insight into the holistic self: from barrier to facilitator; and, the immediate change from unnecessary distraction to mindful engagement. Findings challenge previous conclusions regarding the inability of telehealth to support meaningful relationships, and instead provide novel insights to explain why enabling rural palliative care patients to access support from home is supportive for their wellbeing and the quality of healthcare relationships. Our conclusions question whether the indirect benefits of telehealth could also offer a valuable way of accessing health services beyond a palliative care setting.

Correspondence: Rachel Rahman, Psychology Department, Aberystwyth University, Aberystwyth, SY23 3UX, United Kingdom

E-mail: rjr@aber.ac.uk
\end{abstract}

Key words: telehealth, palliative care, interpretative phenomenological analysis, longitudinal experiences.

Acknowledgements: The authors would like to acknowledge thanks to Gudrun Jones, Hywel Dda University Health Board for her contribution to delivering the psychotherapy sessions as part of this project and to the participants who took part. Thanks are also extended to Carys Stevens, Hywel Dda UHB for her clinical support in organizing the research.

Authors' Contributions: RR, JK and JH conception and design; JK data collection, RR analysis and interpretation of data; JH interpretation of data analysis; RR, JK, \& JH drafting and revising of manuscript and final approval of submission.

Funding: This research was funded by the Knowledge Economy Skills Scholarships (KESS). KESS is a pan-Wales higher level skills initiative led by Bangor University on behalf of the HE sector in Wales. It is part funded by the Welsh Government's European Social Fund (ESF) convergence program for West Wales and the Valleys.

Advisory: To note, the data from this article contributes to a separate article (in review) which compares patients' and health professionals' perspectives on telehealth. The research questions and mode of analyses are different in the two articles and no common quotations are presented.

Conflict of Interest: The authors declare no potential conflict of interest.

Received for publication: 13 January 2020.

Accepted for publication: 11 May 2020.

This work is licensed under a Creative Commons Attribution NonCommercial 4.0 License (CC BY-NC 4.0).

${ }^{\circ}$ Copyright: the Author(s), 2020

Licensee PAGEPress, Italy

Qualitative Research in Medicine \& Healthcare 2020; 4:31-42

doi:10.4081/qrmh.2020.8821

\section{Introduction}

The UK National Institute for Clinical Excellence ${ }^{1}$ defines Palliative care as the active holistic care of patients with advanced, progressive illness (p. 20). This can include pain and symptom management, and psychological support, with the aim of improving quality of life for patients and their families. Whilst palliative care will often include supporting patients at the end of life, some patients may access palliative care earlier in the trajectory of their illness, in conjunction with other treatments. Individuals accessing palliative care increasingly wish to be cared for within their communities and with the opportunity to die at home when the time comes. ${ }^{2}$ In areas where specialist community palliative care or hospice at home teams exist the occurrence of individuals dying out of hospital (84\%) far exceeds the national average in the UK $(52 \%) .{ }^{3}$ However, in rural areas delivery of community care can be challenging as services face higher costs as a result of lower economies of scale, challenging recruitment and retention of specialist staff, difficult geographical areas to service and increased rates of older adults in need of specialist services. ${ }^{4,5}$ It is perhaps not surprising therefore that evidence from rural areas has previously identified that palliative care patients report less symptom control, a lack of regular access to nurses and a lack of communication with medical professionals in comparison with their urban counterparts. ${ }^{6}$ Other studies have highlighted the need for improved access to social and psychological support for palliative care patients. ${ }^{7}$ This is particularly evident in rural areas of the UK, where cur- 
rent models of care have centralized specialist support to urban tertiary centers.

Telehealth involves the use of telecommunications and virtual technology to deliver elements of healthcare and offers a potentially interesting opportunity to improve access to services and support for rural palliative care patients and their caregivers, without the need for unnecessary travel for either the patient or healthcare professional involved. ${ }^{8}$ Kidd et al. ${ }^{9}$ conducted a review of telehealth use in palliative care within the UK and identified a variety of applications including videoconferencing to facilitate multidisciplinary team meetings, telephone help lines, electronic patient records and mobile symptom recording. The review concluded that with adequate infrastructure and support, telehealth offered a feasible and practical mode by which to support traditional models of palliative care in rural and remote communities.

However, Demiris et al. ${ }^{10}$ state that although telehealth is becoming widely used in other health domains it has yet to be adopted as frequently in the provision of palliative care. Their findings suggest that telehealth has the potential to be highly effective in this context given the demonstrable improvements in psychological outcomes seen in other health domains.

More recently, a systematic review of the use of telehealth in palliative care, from the perspective of caregivers, agreed that telehealth interventions were feasible and that users were satisfied. ${ }^{11}$ Amongst articles measuring wellbeing outcomes the benefits of telehealth services included improvements in carers' quality of life, anxiety, depression, stress, and caregiver burden, demonstrating the potential benefits that telehealth connections could offer. However, the authors echoed the concerns of Demiris et al. ${ }^{10}$ regarding the small proportion of telehealth studies conducted in the context of palliative care and highlighted the need to better understand how interventions can support patients during this vulnerable time.

Telehealth studies conducted with patients themselves using quantitative approaches have demonstrated a wide variety of benefits of telehealth services including patient satisfaction, improved symptom control and a reduction in the use of emergency services, ${ }^{12,13}$ increased frequency of contact between patient and professionals, ${ }^{14}$ improved quality of life, and, decreased anxiety and depression. ${ }^{15,16}$

It is clear therefore that the use of telehealth in palliative care can potentially support positive patient outcomes; however, these quantitative studies do not consider why or how telehealth facilitates these outcomes. Understanding the experiences of patients, and the features of telehealth that support or challenge positive outcomes is key to improving services and achieving high quality care for rural residents.

Stern et al. ${ }^{17}$ conducted a case study of remote monitoring and videoconferencing in palliative care which offered some initial insights into the experience of patients through a combination of quantitative, qualitative and ob- servation methods. Findings identified themes around enhanced access to care and usability of the telehealth system. More in-depth qualitative research by Roberts et al. ${ }^{18}$ and Johnston et al. ${ }^{8}$ reports increased patient empowerment though improved opportunities for face to face conversations and involvement in decisions relating to their care. It is possible to see therefore that there are underlying benefits afforded by telehealth technology for rural palliative care patients; however, there continues to be a need for in-depth understanding of how palliative care patients make sense of their experience of using telehealth in rural service delivery over time.

A terminal diagnosis can result in changeable symptoms, emotions and challenges. As such, patient engagement and their needs for telehealth services may vary as patients near the end of life. Similarly, research exploring acceptability of technology suggests that age and experience are key moderators for technology acceptance..$^{19}$ Thus, giving voice to palliative care patients as they navigate these changes during the progression of their illness is key to better understanding the role that telehealth can play in supporting their needs. It is acknowledged that longitudinal research in the context of palliative care can pose challenges due to sample attrition resulting from mortality, increased ethical constraints, and concerns about over-burdening vulnerable participants. ${ }^{11}$ Consequently, the voice of the palliative care patient can be left unheard and their needs and experiences replaced by the observations or opinions of caregivers and family. This poses its own ethical questions about palliative care patient opportunities to engage in their own service improvements.

This study is one of the first articles that takes a longitudinal phenomenological approach with palliative care patients using telehealth and we ask the research question: how do palliative care patients make sense of their experience of using telehealth to access psychological support over a three-month period?

\section{Materials and Methods}

\section{Design}

We utilized an interpretative phenomenological perspective in order to understand how participants made sense of their experience of using technology to access support over time. Interpretative phenomenology provides a method to explore in detail how individuals make sense of their personal and social experiences. ${ }^{20}$ The ability to follow an individual through the novel experience of accessing their service via telehealth, whilst being able to examine their sense making of their own illness and needs during this time, made this approach particularly relevant to this context. We used semi-structured interviews conducted with each participant at three time points following the introduction of telehealth. These occurred at monthly 
intervals. Semi-structured interviews are considered a strength in interpretative phenomenological research because of their ability to offer flexibility, build rapport and enable the participant to lead the direction of conversation, resulting in richer descriptions and sense making of their experience. ${ }^{20} \mathrm{~A}$ longitudinal approach allowed us to better understand how interaction with the technology may change over time. Research has indicated that experience of technology can influence engagement and acceptability, ${ }^{19}$ and as such, we considered the need to allow adequate time for participants to develop experience of using the technology and to understand the change from initial adoption to experience important. However, we also needed to consider the timeframe ethically and practically in light of the palliative care context to not overburden participants and to take account of prognosis. As such, we considered a three-month time period to be in line with previous recommendations for patients to develop a level of experience, with monthly intervals enabling insight into the gradual process of adapting to the technology without intruding on patients too frequently. ${ }^{21}$

\section{Telehealth system and service intervention}

Prior to the research the psychosocial service was only available as a face to face service for patients. They could access the service if they were able to travel to the local general hospital or in cases deemed necessary by the therapist, the therapist would drive to the patient's home. In this rural location, this could involve a journey of over an hour by car and far longer by infrequent public transport. This therefore limited the number of patients who could realistically access the service and the need to improve accessibility for rural patients was identified by the clinical team. We (the research team) were responsible for sourcing a limited amount of equipment to support the initial set-up of the telehealth service and as such, only individuals who consented to participate in the research were able to access the telehealth service at this time. All other patients were able to continue to access the therapist service as per usual care.

The telehealth system used Polycom RealPresence videoconferencing software. This software was installed on laptop computers which were provided for the duration of the study. The software could be used to access a remote VPN which enabled access to a secure network, ensuring a secure connection between the patient's home and the health professional located in the general hospital. Sessions were run at timings deemed appropriate by the therapist and patients, but typically, were once every week to 10 days (between 8-12 sessions over the 3 months) and consisted of one to one consultations between the patient and an art therapist, that lasted up to one hour, as determined by the health of the patient.

The therapist was a registered art psychotherapist with over 20 years of experience but no prior experience of using telehealth to conduct sessions. We (specifically JK) pro- vided technological support and training for both the therapist and patients as required. Participants were provided with a basic supply of art materials and were able to show and discuss their drawings on screen during the session. Art therapy sessions were used to encourage participants to share their current experiences, explore their feelings towards their terminal diagnosis and consider ways to alleviate or resolve any concerns. Reporting on the experiences of the therapist is beyond the scope of this paper; however, the therapist's reflection on developing a telehealth-based art therapy service has been published as a source of reference and advice for other practitioners. ${ }^{22,23}$

\section{Participants}

Participants were approached by members of the multidisciplinary team following multidisciplinary team meetings where potentially suitable patients were discussed. Inclusion criteria included patients who were recipients of specialist palliative care and who lived in a rural location (determined as living a minimum of 45 minutes' drive from the local palliative care unit). Exclusion criteria included those under the age of eighteen, lacking capacity to provide informed consent, or who did not possess a working internet connection. Members of the multidisciplinary team were initially hesitant to discuss the project with patients as a result of concerns about the ability of patients to cope with additional demands of research and learning new technology. However, over time a number of suitable patients were identified and approached by members of the multidisciplinary team who provided an overview of the service and research. Patients who expressed an interest were provided with a participant information sheet and reply slip which could be returned to us (the research team). To our knowledge, all individuals who were invited to participate opted to do so except for one patient who did not have an internet connection at home and so was unable to be recruited to the study.

We recruited an initial sample of four palliative care patients ( 3 female, 1 male) ranging in age from 48 to 72 years. All of the participants had been diagnosed with inoperable terminal cancer of different primary locations; however, they were not all necessarily at the point of end of life care (see Table 1). One participant died following the first stage interview. The data collected through this interview were removed from the main analysis because of the inability to consider longitudinal sense making. The remaining three participants completed all three interviews. To maintain anonymity, the pseudonyms Peggy, Coleen, and Jackie have replaced participant names. Two of the participants (Peggy and Jackie) had been receiving face to face psychosocial support in the general hospital and were therefore changing to access their care via telehealth. The other participant (Coleen) had previously been unable to access the face to face service due to travel difficulties and was therefore accessing her psychosocial 
support for the first time. A summary of their profiles is provided in Table 1.

\section{Procedure}

We gained ethical approval for the study from the NHS research ethics and Research and Development committees of the local health board. We visited participants at home where they provided written informed consent and received a face-to-face demonstration of the telehealth system. We provided written instructions and asked participants to repeat the demonstration independently to ensure they understood how to make the connection. We conducted monthly semi-structured interviews in the home of the participant on a one to one basis. Interviews were audio recorded for the purpose of transcription and asked participants about their experiences of using the system to access their support. The interview schedules were informed by previous literature exploring technology adaptation in other contexts. ${ }^{19}$ The first interview focused on previous experience of technology, experience of learning to use the system and considered benefits and limitations to the participants' experience (for example: How did you find getting used to the equipment? How comfortable were you discussing your needs through the medium of telehealth?). Additional prompts were added to the second and third interview schedules to specifically ask about whether or how their views or experiences had changed since the previous interview (for example: Has your experience of using the telehealth to access support changed in any way from previous interviews? Have you noticed any changes in the way that you are using the equipment in comparison to previous months?). Interviews ranged in length from 21 to 79 minutes depending on the patient's health on the day of the interview.

\section{Data Analysis}

Each of the semi-structured interviews was transcribed verbatim using a professional service. We used longitudinal Interpretative Phenomenological Analysis (IPA) to consider individual experiences over a three-month period. Whilst IPA is a well-known qualitative methodology for understanding lived experiences in health, ${ }^{24}$ longitudinal IPA continues to be a novel approach to understanding temporal subjective experiences, and, methodological frameworks to guide analysis are limited. ${ }^{25}$ Smith and Osbourn ${ }^{26}$ suggest that when using IPA it is advisable to begin analysis by looking at one case study in detail before moving on to ex- amine the others. Due to the longitudinal design of this study, this advisory note was extended to look at each individual time-point interview in isolation before considering across time for each individual and later across cases. The analytical process involved using a double hermeneutic approach focusing on how the participant made sense of their experience before commencing our own interpretative process. I (first author) was responsible for coding and theming the data at an individual level across time and for the development of the overarching themes that illustrated the longitudinal process across participants. My co-authors reviewed coded extracts and themes to triangulate perspectives and contributed to interpreting the underlying sense making and interpretations that were being voiced by participants. We adopted Farr and Nizza's ${ }^{25}$ recommendations for reporting findings with consideration of both the longitudinal and cross case aspects of the data. This allowed greater insight into the meaning behind the respondent's sense making at a specific time point, and whether this changed during their three-month involvement in the research project.

\section{Results}

We identified four superordinate themes that represented the temporal changes relating to telehealth use across cases and time points. These were titled: Deepening understanding through unburdened and continuous connections; the ever-present paradox of visible and invisible telehealth; insight into the holistic self: from barrier to facilitator; and, the immediate change from unnecessary distraction to mindful engagement.

\section{Deepening understanding through unburdened and continuous connections}

Feelings of isolation either through illness, remoteness or disconnect were a key focus for all participants who sought opportunities to develop meaningful relationships with people and their environments. Participants were concerned about burdening family and friends with their diagnosis and worries; however, the continuity of the relationship with the therapist over time led to the development of a deeper sense of understanding of their concerns. The ability to do this with a trained professional whose role it was to support them, also alleviated them of concerns about the burden they were placing on the shoulders of another.

Table 1. Patient demographics and profiles.

\begin{tabular}{lcccc}
\hline Participant indicator & Age & Gender & Cancer Diagnosis & Living situation \\
\hline Peggy & 48 & Female & Breast & Alone \\
\hline Coleen & 63 & Female & Lung & Alone \\
\hline Jackie & 72 & Female & Bone & Recuperating in a care home \\
\hline
\end{tabular}


Jackie was temporarily recuperating in a care home away from her friends and family. In the first interview, she discussed a lack of connection between herself and the carers, who she regularly referred to as friendly but very busy. She referred to feelings of isolation from being away from friends and family. By the end of the first month the connection that Jackie had developed with the therapist during telehealth consultations had already started to create a consistent sense of relatedness that offered an outlet to discuss her concerns.

Well, it's just nice to have somebody to talk to. I think the most important thing is it's nice to have someone that cares, what you think and how you're feeling which because obviously my family are all away, I don't have anyone to ask how I am or what you're feeling. Everybody is too busy. [Jackie; Month 1].

As time progressed, Jackie became more independent and required less care from residential staff; however, the isolation of the care home and a disconnect from an enriching environment appeared to limit Jackie's feelings that she was able to connect in the same way during her consultations. The feeling that her experiences failed to change from day to day limited the variation of the conversations that she felt able to have.

I haven't got lots to talk about to be truthful,

being in one room in a home. Not much happens.

So, there's not a great deal to talk about really.

[Jackie; Month 2].

By the final time point, Jackie had become well enough to move to supported housing where she could live more independently. As the potential to develop better connections outside of the care home became a possibility, so did her invigoration for conversation with the therapist. Jackie recalled previous experiences of having close connections with friends to the point that they could identify each other's concerns, despite them not being articulated. Jackie drew comparisons with the relationship that she had developed with the therapist who could provide a balanced view but with a degree of personal insight to her situation, thus suggesting that they had been able to form a close connection in a similar manner.

Yeah, it's just having a talk with someone really and it's just like knowing that there's somebody there that they know what's going on and sometimes - because in my experience, when I have a friend that's going through a problem, I think sometimes they pick up things that you don't really know is happening. So, sometimes I feel I'm down but then I get talking, well I'm not too bad. [Jackie; Month 3].

Coleen's ill-health had recently resulted in her having to leave her much loved cottage in her local community to move to a more modern apartment. The disconnect from her previous home and friends had left her feeling isolated and lacking support and the feeling of not want- ing to burden friends with her worries about her terminal illness were evident across the three time points. The telehealth connection enabled her to build a consistent and supportive relationship with someone who was trained to cope with the difficult conversations that Coleen needed to have about her terminal diagnosis.

The friends I've left it's a bit upsetting, you know, because I can't see them and I haven't really wanted to talk to them on the 'phone because I've had no energy or I've just been so ill I thought nobody wants to hear anything I've got to say at the moment, I wouldn't impose it on anyone. So that's been good having [therapist's name] because she's sort of obviously trained to deal with - like your friends aren't trained to deal with all the stuff you sort of go through. [Coleen; Month 1].

Coleen's connection with the therapist developed into a mutual understanding that despite a cheery persona the concerns and experiences continued to exist under the surface. It was apparent that the quality of the relationship that developed remotely with a trained professional provided a deeper connection to enable Coleen to share aspects of her life that she wanted to protect from friends and family.

I feel better that I've - that she can deal with it, although the people I talk to I wouldn't say anything I didn't think they could deal with, but then a lot of people they sort of say, but you always look so cheerful and he [neighbor] said you look well, he looked cheerful and I suppose talking to [therapist] she can see that, but she knows there's big issues going on which I can explore more with her [Coleen; Month 2].

By the final time point, Coleen reflected on the fact that her long-term relationship with the therapist had resulted in a deeper understanding of her progress. Insight into her changeable feelings came without the need to hide her true emotions in the way she felt she needed to with others.

I imagine [therapist] expects the whole thing to be more of a process in you are going to churn things over and then come back at a later date and think well that's how I was feeling then, but I feel like this now (...) So it's quite good I suppose that she can follow your progress through the treatment and probably have more of an understanding of what you're going through than sort of people you see every day [Coleen; Month 3].

Unlike Coleen and Jackie, Peggy continued to live in a small rural village amongst her community. For her the need for a connection was less evident in her interviews, suggesting that her needs for support were being met outside of the service being offered. However, on the few occasions that Peggy discussed the nature of her relationship with the therapist, the role of the environment appeared a key feature. At time one, Peggy discussed a feeling of dis- 
tance when meeting over telehealth; however, having previously been in the therapist's room, being able to see into a familiar environment helped to draw this connection closer and create a feeling of being present.

The difference is, that I suppose is that slight distance feeling of, you know, you are looking at somebody on the screen although it does kind of look really real (...) But because you know the room and you've been in there, the same sort of space, then kind of I suppose it does feel like you're really there. [Peggy; Month 1].

The importance of visualizing the other person's physical location re-emerged for Peggy at month 3, but this time from the other person's perspective. For Peggy, the fact that the therapist could visualize her environment created a feeling of inviting her into her home. This sense of sharing an environment, albeit virtually, helped to form a closer connection, and in a similar manner to Jackie and Peggy, elevated the therapist to a status of being a friend.

I think because like on the teleconference they're in your home, you kind of feel more familiar with them. Whether that's a good or bad thing with a therapist, I don't know, but you kind of feel more like they more fall into the category of your friends because obviously they're coming into your home [Peggy; Month 3].

\section{The ever-present paradox of visible and invisible telehealth}

The changing visibility of the technology within the physical and virtual environment emerged as a relevant theme for participants, but the manifestation of this varied across the three time points rather than changing as a process. All participants, at varying times, discussed how the distance created by the technology made them less visible which facilitated a freedom to talk. However, at other times being consciously aware of the technology also served a meaningful purpose, creating focused conversation, acting as a comforting reminder of available support, or creating a protective shield to the visibility of their emotions.

Both Coleen and Jackie started the process as self-described technophobes, claiming to have little experience or confidence with technology. Despite this, Coleen discussed how she quickly lost the conscious awareness of being in conversation through a machine; however, she was surprised when the conversation reminded her that the person on the other side of the screen had a view of her. This suggests that in a short space of time, the technology became invisible to Coleen and the conversation felt natural.

I think it did (feel natural) almost straight away. I just forgot that I was talking to a machine. So but - yeah, I don't - it's gone now. I don't know. I think almost instantly I, it seemed to become fairly natural apart from when she said don't forget your tea and made me jump. [Coleen; Month 1].
However, over time the computer became visible as an extension of the shared virtual space. The physical presence of the technology acted as a reminder of her opportunity to discuss things that were worrying her; enabling her to mindfully place concerns to one side until a future appointment and providing a symbolic connection to the source of support.

Well, in every way it's just quite amazing having a screen and somebody (laughter) I don't know. Just because $(. .$.$) well so you have it in your mind. That$ will be my slot when I can discuss all my problems and everything so (...) you can just sort of think well, okay, I'll put those in a box and I can talk about it with [therapist] rather than having it going around and around. [Coleen; Month 2].

Interestingly, when contrasting her own initial assumptions that a face to face relationship would be superior, Coleen reflected that the physical technology acted as a shield between her and the person on the other side of the virtual divide. For Coleen, the lack of physical proximity offered a protective barrier that enabled her to feel more able to express her emotions:

Yeah because you might think well, okay, that's easier [being face to face] because I can unload myself more although I'm still doing it to [therapist], you think it's sort of a shield sort of thing whereas it might be different if I'm [in person]. [Coleen; Month 2].

However, in the same interview, the invisibility of the technology re-emerged when she discussed the benefits of the distance afforded by the technology and reflected on her continued ability to disclose more personal information than she would have done in a face to face interaction. Coleen believed that the honesty that was developed in the virtual environment allowed the therapist a better insight to her true self.

Well she might even know me more from doing it like that from face to face really. (...) So probably I've just disclosed or talked about more than I would have done in real life, really. [Coleen; Month 3].

Jackie required support from care home staff to set up the technology ahead of consultations, but she also adapted quickly to the telehealth system. By the end of the first month she was discussing the benefits that being on the other side of the screen afforded her in terms of expressing her feelings. Whilst being able to visualize the therapist, Jackie also discussed being on her own as though the distance created a comforting invisibility from the therapist, enhancing her perceived ability to express herself.

And I think also, it sounds silly but being in a room where you're on your own, you tend to talk more because you feel that there's just nobody there. [Jackie; Month 1].

By the second time point this distinction between face 
to face consultations and the telehealth system appeared less apparent and Jackie saw the two as directly comparable in terms of the quality of interaction, suggesting that the distinction between what was visible or invisible, present or remote, became less important.

It doesn't make any difference to me whether she comes to see me in the room or the computer.

As I've said, I just have a talk with her and that's it really. So, it doesn't make much difference at all. [Jackie; Month 2].

In the final interview, Jackie continued to see no difference in the quality of interaction; however, she returned to reflecting on the benefit of being able to enter a space where the therapist was not visible and her experience became aligned to talking to oneself. It appeared therefore that the ability to choose whether the therapist was visible or not created the freedom to just talk.

To be truthful, I don't find any real difference. I just sit down and talk. Sometimes, I don't even look at [therapist name] I just talk either way and I think if anyone could see me sat in this room talking to myself — no, but I find it very good, yeah. [Jackie; Month 3].

Peggy had experience of using computers for the purpose of emails and surfing the internet; however, had never used videoconferencing. She had previously been accessing support from the therapist face to face and she reflected on this comparison. She acknowledged the impersonal nature of the technology, however, for Peggy, the visibility of the therapist enhanced the intimacy and focus of the interaction.

I suppose one thing cancels the other one out in that respect because you are looking at a screen at the end of the day, so it's probably more impersonal in that way. But then because you are very much focused one-on-one and there are no other distractions, then yeah, I kind of think it does, probably cancels it out really. [Peggy; Month 1].

However, the unfamiliarity of the interaction was evident and potentially enhanced by the contrast of the perceived intimacy and the cold closing of the interaction at the end of a consultation. For Peggy, the need to 'switch someone off' gave an awkward abruptness to the move from visibility to invisibility of the therapist.

That is kind of a bit weird really because you kind of like - you kind of wrap it up, but normally in that case you're going to move in that room and you're still together. Whereas you feel like you're turning somebody off, you know [Peggy; Month 1].

Whilst the central part of the consultations became familiar and natural for Peggy, enabling her to forget the remoteness of the consultation, the instant visibility and later invisibility at either end of the consultation continued to feel alien and uncomfortable.

I think the initial start is a bit uncomfortable for me still, when you first sort of ping and you see each other and you're sort of — but when I do get into it I do kind of relax more and kind of forget more that I'm actually on the conference. And then at the end, again it can feel a bit strange I think because you're kind of a bit like sometimes when you're on the phone, like you hang up first, no I'll hang up first (laughing) [Peggy; Month 2].

In a similar manner to Coleen, by the end of month 3 , Peggy reflected on the symbolic role that the visibility of the technology played for her by extending the shared virtual space and acting as a reminder of the support that was available to her.

I think maybe from a psychological point of view it's having the equipment in your house makes you feel you've got that connection. Whereas when you come here [hospital] and you go home, it's like you don't have any connection do you? I mean, I know it's only a computer in your room, but it does feel I suppose that you've got that constant connection if you need it [Peggy; Month 3].

\section{Insight into the holistic self: from barrier to facilitator}

The visual element of videoconferencing was a feature noted by all participants. Sense making of the self was initially stilted by being aware of the physical image on screen, this created feelings of self-consciousness and removed the naturalness of the conversation. This became less relevant for different participants at different time points; however, as time progressed this consciousness was replaced by an increased awareness of the benefits that came with the therapist being able to see them. The ability of the therapist to read their emotions and be aware of their own physical space helped to create a sense of being a person within a context, in contrast to a patient in a hospital.

For Coleen, being able to see someone, and be seen, held them more accountable in the conversation and ensured a focus being afforded to one another.

I feel that when you're talking on that that you're (pause) — I don't know, that you have more a sense of responsibility in the conversation somehow because she can see someone. [Coleen; Month 1].

The ability of the therapist to see Coleen in her own home or being able to show pictures that she had drawn of meaningful places also created a sense of improved understanding and awareness of her holistic self. Being able to convey to the therapist where she chose to spend her time and have insight into her life appeared to be an additional way to reflect her identity.

And I mean, that's amazing because you couldn't do that on the 'phone really and [therapist name] can probably understand me better because I can say this is where I choose to spend my day, this is what I like. [Coleen; Month 1]. 
The awareness of her own image only became apparent to Coleen at time 2 where she discussed the dislike of being able to see herself. She also reflected on this at time 3 discussing how her own image distracted from the conversation. However, she had been made aware of how to hide this element, enabling her to avoid seeing a constant reminder of herself.

It's a bit alarming when you come in and see yourself on there, I don't mind seeing [therapist] but I don't like it when I'm up on the screen. [Coleen; Month 2].

However, by time point three the benefits of being able to see each other had more of a focus for Coleen; again, she discussed how the added visual communication enabled an increased ability to read someone and better understand their underlying emotions.

Oh, I think that's better on the screen if you just the fact that I could show her drawings. It's more strings to the bow, isn't it, they can get more idea than on the telephone (...) Because you can quite disguise it on the telephone, but you can't really when someone can see you. [Coleen; Month 3].

The visual images of the self also added a sense of self-consciousness to the interaction for Peggy. She discussed feeling suddenly aware of her appearance through the portrayal of her own image on the screen and this acted as a distraction to the deeper sense making that the therapy encouraged. However, she discussed how over time the initial focus on the image of herself became minimized and faded to the point of disappearing.

You can see yourself as well, which is kind of - because normally if I was there in the room I wouldn't be able to see myself. But because you've got that little picture of yourself on the screen as well, you suddenly realize you're slumping, sit up straight and my body posture is terrible. You kind of get a little bit distracted from that initially, but as you get used to using it you kind of don't look at that little picture of yourself; that kind of just seems to disappear even. [Peggy; Month 1].

At month 2 Peggy continued to reflect on this and attributed her discomfort with her image to the unfamiliarity of the technology as a mode of conversation, comparing it to the familiar feeling of communication without visual input.

I feel a little bit self-conscious when it's faceto-face on the screen. But again, I think that's just time because I don't use teleconferencing for anything else [Peggy, Month 2].

However, despite this unfamiliarity, Peggy appreciated the benefit that the added visual ability could have for the therapist, enabling her to gain a better understanding of Peggy's emotional wellbeing through these cues.

I would think probably from her point of view the teleconference would tell her more where you stood emotionally and stuff because obviously you've got that added dimension of being able to see the person's body language and how they are.

[Peggy; Month 2].

The unfamiliarity of the visuals of the self were slowly becoming more familiar to Peggy by time point 3; however, she continued to dislike seeing herself on screen. She had become aware of the ability to remove this screen from the visual scene portrayed and felt that with continued use the prominence of the self-image would be minimized in importance.

I think slowly I'm getting used to that, slowly. It's still a bit strange I think. But it's that whole thing isn't it when you see photographs of yourself, you're like ooh. And it's like constant you've got a photograph of yourself staring back at you, although you can turn it off...Yeah, I mean it's kind of okay I think over time you'd kind of block it out. It would just become something that you didn't kind of think about, I think probably over time. [Peggy; Month 3].

Jackie's initial experience was different from the other two participants' experiences. Jackie described being conscious of others seeing her visual expressions when communicating face to face about emotive subjects. However, when communicating remotely via videoconference, Jackie appeared to be less concerned about the therapist's ability to see her. It appeared that the distance created by the technology removed this self-consciousness for Jackie, enabling her to feel more able to express herself.

So, I'm talking to her but I talk - if you're face-to-face with someone you have to hold back a lot, because obviously they can see your reaction.

So, when I went at the computer, I just got along.

[Jackie; Month 1].

Interestingly, in comparison to the other participants, Jackie made no reference to the visual aspects of the consultations at any time points after this, and by the final time point only reflected on the strength of her relationship with the therapist and the ability to have meaningful conversations. This might either suggest that the visual element of videoconferencing had no significant role for Jackie, or that the images appeared adequately natural such that this was no longer a notable feature for her.

\section{The immediate change from unnecessary distraction to mindful engagement}

The need to travel to consultations was a considerable burden for these rural patients. From as early as the first month of the telehealth service, participants discussed the benefits of being able to remove unnecessary practical worries. This created space for participants to engage with their own therapy in a more mindful way and facilitated more autonomy about how they spent their own time. For Coleen, the telehealth connections removed the pressure to involve other friends or family to drive them to consultations and alleviated concerns about parking at the hos- 
pital. The removal of this additional stress and anxiety enabled Coleen to achieve more focus in her sessions.

It's really nice being able to do that [talk to someone] without having to make a journey and because then when you do that you're more focused on the road and the getting there and the traffic lights or you know, parking. And this you can just turn up and do what the actual thing is about rather than having to be side-tracked in all these other experiences. [Coleen; Month 1].

Coleen continued to draw this comparison between the hospital environment and her ability to connect from home. The hospital contributed additional worries and concerns that fed into consultation time and she became preoccupied with these concerns preventing her from focusing on the deeper psychological concerns that she may have been experiencing. For Coleen removing this unnecessary anxiety allowed a more focused discussion with the therapist.

Because by the time we've done the journey and gone into different environment, hospital, you're taking on all that, aren't you?...Yeah, then when you go to talk about it you're probably more likely thinking about the problems you've had on the journey rather than the problems going on in your life. So I think this is much more immediate like that. [Coleen; Month 2].

In the final interview, Coleen focused less on the anxieties of the hospital environment and focused more on the risks of chemotherapy induced immuno-suppression. She was mindful of the need to avoid too much contact with people who could carry infection and considered the telehealth consultations a way to ensure continued support but without the risks to her health.

...when you're having chemotherapy, you're told to avoid people all over the place, so that is actually a really good opportunity isn't it, to have someone to talk to that you're not going to catch a cold. [Coleen; Month 3].

Peggy also saw the removal of the unnecessary travel concerns as an opportunity to better prepare for and reflect on her sessions. She used the additional time to take notes before and after her consultations thus feeling she had space to achieve more sense making from her therapy

I think the other advantage of doing it from home as well, is that after the session if you need to make any notes or you just want to sit quietly and think about what's been said or do some work on what you've done in the session, you can obviously do it straightaway. Whereas if you're in town you've got to drive home and then you've forgotten. [Peggy; Month 1].

This continued to time point 2 where Peggy would reflect on what she wanted to get out of sessions more mindfully. Being on your own ground also created a sense of confidence, enabling her to take a more active role in her consultation and direct the conversation to her own agenda.

And usually they say something like, okay is that it? or, are you happy? or whatever and, you know, if you're at home on your own ground it would give you that bit more confidence to say I need to ask you this. [Peggy; Month 2].

The burden of attending hospital appointments continued to be evident for Peggy in the final interview. Peggy discussed how travelling to appointments dominated a full day, causing fatigue and wasted opportunities. Peggy reflected that connecting from home freed up her day enabling her more opportunities to engage with the things that she enjoyed in her life.

Being able to stay at home (...) like I've come in today to [the hospital] it's been - that's what I've done for the whole day, if you know what I mean (...) But, you know, if I was at home that would have taken an hour of my time at home, if you know what I mean, and I'd probably of $\{$ sic $\}$ had the rest of day to do what I want. So, I think that's probably the main advantage. [Peggy; Month 3].

Jackie was not connecting from her home environment and instead was connecting from the nursing home where she was recovering from treatment. There was therefore limited discussion about the benefits of the home environment for her as she convalesced away from home. Despite this, at the first-time point Jackie discussed how the added privacy of being in her own room within the care home, away from the proximity of the health professional with whom she was connecting was key to her ability to speak freely and honestly about her condition.

To be truthful, as I said, I think it's easier for me to talk, even though I can talk to - you know, but I just feel like I said before, you feel that you're on your own, the door is closed. No one can listen or hear you. So, I think I tend to talk probably a bit more. [Jackie; Month 1].

\section{Discussion}

In this study, we aimed to address the research question: how do palliative care patients make sense of their experience of using telehealth to access psychological support over a three-month period? The participants represented individuals of varying ages, all with cancer diagnoses of varying types and who lived in rural locations that created challenges accessing regular support from their palliative care team. Experiencing their illness within a rural context had resulted in individuals being unable to access formal psychosocial support due to a lack of service provision in their county or had resulted in them having to leave their communities to access their care more readily, or to recover from treatment. These left individuals feeling isolated from their communities. Shaw et al. ${ }^{27}$ discussed the importance of feeling rooted to a place for 
wellbeing needs. The upheaval and isolation experienced by each of the participants clearly challenged this connection and telehealth offered an opportunity to reconnect to sources of support.

Our longitudinal approach enabled a better understanding of the process that participants went through as they adapted to the technology and engaged remotely with psychotherapy. Some of the experiences changed immediately within the first month of the service and continued throughout the three-month period, such as enabling individuals to avoid unnecessary practical distractions and develop a more mindful engagement with the psychotherapy service that they were offered. This created room for reflection and a focus on the self that appeared to have been reduced when receiving face to face care in the hospital. Literature and theoretical approaches regularly discuss how frequently patients forget consultation information or feel too intimidated to ask questions; in consequence limiting patient satisfaction and/or compliance. ${ }^{28}$ Similarly, theories such as self-determination theory ${ }^{29}$ discuss the importance of autonomy supportive environments where patients are supported and encouraged to question and engage in health-related decisionmaking. The experiences of individuals connecting from their home environment suggests that the context could provide a valuable means of addressing some of these features for some patients, thus empowering them to engage more openly with the management of their palliative care.

Other experiences were continually fluctuating. For example, the conspicuous visibility of the technology and therapist regularly changed as participants discussed their experience. The therapist could be visible or invisible and this served to enable the participant the choice to feel alone and private, but also supported. The technology could be consciously visible creating a protective distance or a reminder of the continuity of the available support, or it could create a sense of being invisible, facilitating a natural conversation. The additional visual stimuli afforded by telehealth enabled enhanced communication through body language or visual expression and created a sense of shared space and autonomy, where the participant could choose to invite the therapist into their home. This was perceived to develop a more personal connection through a better understanding of their intimate feelings and their holistic lives. Previous research has indicated the importance of non-verbal communication in the manifestation of emotion and patient satisfaction. ${ }^{30}$

Other experiences were described more as a process of development. The developing relationship between the participants and the therapist was evident as the relationship became increasingly insightful, resulting in the relationship being described in terms of friendship. Despite this, participants articulated the difference between their friendships and the therapeutic relationship which placed their needs at the centre of conversation and removed concern of burden. It was evident through each patient's ex- perience that individuals were able to develop strong and meaningful connections with the therapist despite the physical remoteness. As such, findings of this study contrast the proposed limitation that telehealth could cause disruption to the therapeutic relationship. ${ }^{31}$ Instead, findings are supportive of prior studies that have demonstrated the benefits that telehealth provision can offer for psychosocial support in rural areas. ${ }^{14,16}$ The development of these effective therapeutic relationships was built on the affordance of time dedicated to the relationship, the freedom to discuss burdensome topics without guilt, and the intimacy of personal knowledge gained through the communication. In later themes the relevance of the telehealth as a supporting rather than thwarting factor for these developments was evident.

Interestingly, alongside the development of the therapeutic relationship appeared to be a development of an increased sense of self. Concerns about physical appearance on screen appeared to develop towards an awareness of the deeper sense making that could be created when the therapist shared the patient's personal space, but at a safe distance. This created a sense of presenting a holistic person to the therapist, with a life beyond the hospital and their illness. The importance of assessing a patient holistically has been highlighted, supporting good quality of life and symptom management. ${ }^{32}$ Thus whilst telehealth appears to have advantages for rural communities, we propose that this finding raises interesting questions about the benefits that home consultation could potentially offer more broadly in developing more patient centred care, enabling the health professional to make a connection between the individual and their lives beyond their illness.

Despite the novel understanding we have offered here, this study is not without limitations. As is common and encouraged for IPA studies, ${ }^{26}$ the sample size is small. It is possible that in selecting patients the multidisciplinary team may have inadvertently been biased towards individuals who they perceived to be more open to trialling such a service. As such, the experiences discussed in this study may only be transferrable to those who are open to engaging with telehealth. In addition, whilst reflective of the relatively homogenous population from which they were sampled in rural Mid Wales, we acknowledge that the sample was lacking in cultural diversity and included only individuals with cancer. The scope of palliative care has moved to be more inclusive of other life limiting illnesses, and different cultural backgrounds which may bring with them differing perceptions and expectations. There is a need to consider more broadly what benefits and challenges telehealth might offer individuals with dementia, motor disease or communication difficulties, for example, as well as how acceptable such a service might be to different cultural groups. The interviewer in the study (second author) was also the person who set up and demonstrated the equipment, and therefore despite all documentation being clear that we were independent to 
the service, there is a possibility that patients felt less able to be critical. However, transcripts appeared to demonstrate honest and balanced views.

\section{Conclusions}

In conclusion, our article is one of the first to consider the in-depth longitudinal experience of patients accessing psychological support via telehealth as part of palliative care. Palliative care patients have been under-represented in the literature in comparison to other clinical populations, particularly with longitudinal approaches, resulting in the patient voice being less apparent in palliative care decision making. Whilst the sample is small, the in-depth accounts of these patients provide important insights into why supporting patients to remain at home whilst receiving palliative care is important for their wellbeing, sense of place, and empowerment. Telehealth, as a component of their care, appeared to be an effective method for achieving this, and in contrast to previous literature, demonstrated that meaningful relationships could be created between patients and health professionals despite the lack of physical proximity. We suggest that these patient experiences also raise interesting considerations about how healthcare services are delivered more generally, highlighting how the home environment can, in some situations, support patient empowerment and engagement in contrast to clinical environments. This is particularly pertinent as numerous parts of the world (currently May 2020) face lockdown and reduced face to face care in the current Covid-19 pandemic. Despite the obvious practical, ethical and legal challenges that can be of concern when delivering telehealth services, it is comforting to be aware of the meaningful benefits, and patient centred care, that telehealth can potentially offer.

\section{References}

1. National Institute for Clinical Excellence (2004). Guidance on Cancer Services Improving Supportive and Palliative Care for Adults with Cancer. The Manual. 2004;ISBN:184257-579-1. p.20.

2. Marie Curie. Dying at home: The role of social housing providers in supporting terminally ill people in Wales. Policy and Public Affairs Team, Wales, July 2014.

3. National Council for Palliative Care. National survey of patient activity data for specialist palliative care services 201415. Available from: https://www.hospiceuk.org/docs/ default-source/What-We-Offer/publications-documents-andfiles/minimum-data-set-hospital-support-report-201415.pdf?sfvrsn=0. Accessed: January 2020.

4. Deaville JA. Health-care challenges in rural areas: physical and sociocultural barriers. Prof Nurs 2003;18:262-4.

5. Strasser R. Rural health around the world: challenges and solutions. Fam Prac 2003;20:457- 63. doi: 10.1093/fampra/ cmg422.

6. Welsh Assembly Government. Rural health plan; Improving integrated service delivery across Wales. Welsh Assembly government report 11:19:2009.

7. Hughes PH, Ingleton MC, Noble B, Clark D. Providing cancer and palliative care in rural areas: a review of patient and carer needs. J Palliat Care 2004; 20:44-9.

8. Johnston B, Kidd L, Wengstrom Y, Kearney N. An evaluation of the use of telehealth within palliative care settings across Scotland. Pallit Med 2011; 26:152-61.

9. Kidd L, Wengstrom Y, Johnston BM. Telehealth in palliative care in the UK: A review of the evidence. J Telemed Telecare 2010;16:394-402. doi: 10.1258/jtt.2010.091108

10. Demiris G, Oliver DP, Wittenberg-Lyles E, Washington K. Use of videophones to deliver cognitive-behavioural therapy to hospice caregivers. J Telemed Telecare 2011;17:142-5.

11. Zheng Y, Head BA, Schapmire TJ. A systematic review of telehealth in palliative care: Caregiver outcomes. Telemed e-Health 2016;22:1-7.

12. Hennemann-Krause L. Lopes AJ, Arav́jo JA, et al. The assessment of telemedicine to support outpatient palliative care in advanced cancer. Palliat Support Care 2015;13: 1025-30.

13. Watanabe SM, Fairchild A, Pituskin E, et al. Improving access to specialist multidisciplinary palliative care consultation for rural cancer patients by videoconferencing: report of pilot project. Support Care Cancer 2013;21:1201-7.

14. Olver I, Brooksbank M, Champion N, Keeley J. The use of videophones to enhance palliative care outreach nursing in remote areas. Prog Palliat Care 2005;13:263-7. doi: 10.1179/ $096992605 \times 57679$.

15. Larson J, Rosen AB, \& Wilson FA. The effect of telehealth interventions on quality of life in cancer patients: A systematic review and meta-analysis. Telemed e-Health 2018;24:397-405. doi: 10.1089/tmj.2017.0112.

16. Shepherd L, Goldstein D, Whitford et al. The utility of videoconferencing to provide innovative delivery of psychological treatment for rural cancer patients: results of a pilot study. J Pain Symptom Manage 2006;32:453-61.

17. Stern A, Valaitis R, Weir R, Jadad AR. Use of home telehealth in palliative cancer care: a case study. J Telemed Telecare 2012;18:297-300. doi: 10.1258/jtt.2012.111201.

18. Roberts D, Taylor C, MacCormack D, Barwich D. Telenursing in hospice palliative care. Can Nurse 2007;103:24-7.

19. Venkatesh V, Thong JYL, Xu X. Unified theory of acceptance and use of technology: A synthesis and the road ahead. J Assoc Inf Syst 2016;17:328-76.

20. Smith, JA. Qualitative Psychology, a practical guide to researchers (2nd ed). London; Sage: 2008.

21. Davies A., \& Newman S. Evaluating telecare and telehealth interventions. London: The King's Fund, Department of Health, UK: 2011

22. Jones G, Rahman R, \& Robson, M. Group art therapy using telemedicine technology for immunosuppressed patients undergoing chemotherapy. In M Wood, B Jacobson \& H Cridford (eds), The International Handbook of Art Therapy in Palliative and Bereavement Care. Routledge International Handbooks, Taylor \& Francis: 2019. doi.org/ 9781138087330:

23. Jones, G, Rahman, R \& Robson, M. Group art therapy and telemedicine. In CA Malchiodi (ed.), The Handbook of Art Therapy and Digital Technology. Jessica Kingsley Publishers: 2018.

24. Brocki JM, Wearden AJ. A critical evaluation of the use of interpretative phenomenological analysis (IPA) in health 
psychology. Psychol Health 2006; 21:87108. doi: 10.1080/ 14768320500230185 .

25. Farr J \& Nizza IE. Longitudinal interpretative phenomenological analysis (LIPA) a review of studies and methodological considerations. Qual Res Psychol (online) 2019;16:199-217. doi: 10.1080/14780887.2018.1540677.

26. Smith J, Osborn M, Interpretative Phenomenological Analysis. In: Smith, J. Qualitative psychology: A practical guide to research methods. London, UK: Sage; 2008. pp 53-80.

27. Shaw RL, West K, Hagger B, Holland CA. Living well to the end: A phenomenological analysis of life in extra care housing. Int J Qual Stud Heal 2016;11:1-12. doi: 10.3402/ ghw.v11.31100.

28. Ley P. Improving patients' understanding, recall, satisfaction and compliance. In: Broome AK ed. Health Psychology.
Boston, MA: Springer Sciences; 1989. pp74-102. DOI: 10.1007/978-1-4899-3228-0 5

29. Deci, EL, Ryan RM. Self-determination theory: A macrotheory of human motivation, development and health. Can Psychol 2008;49:182-5.

30. Reiss H, Kraft-Todd G. A tool to enhance nonverbal communication between clinicians and their patients. Acad Med 2014;89:1108-12.

31. Shepherd L, Goldstein D, Olver I, Parle M. Enhancing psychosocial care for people with cancer in rural communities: what can remote counselling offer? Rehabil Disabil Ageing 2008;32:423-38.

32. Maher D, Hemming L. Understanding patient and family: holistic assessment in palliative care. Br J Community Nurs 2013;10. doi: 10.12968/bjcn.2005.10.7.18327 